\title{
Naturally Derived Organic Dyes for LED Lightings of High Color Rendering and Fidelity Index
}

Yunping Huang, Theodore A. Cohen and Christine K. Luscombe*

Yunping Huang and Prof. Christine K. Luscombe

Department of Materials Science \& Engineering, University of Washington,

Seattle, WA 98195

E-mail: luscombe@uw.edu

Theodore A. Cohen and Prof. Christine K. Luscombe

Molecular Engineering \& Sciences Institute, University of Washington, Seattle, WA 98195

Prof. Christine K. Luscombe

Department of Chemistry, University of Washington, Seattle, WA 98195, USA

Keywords: lightings, color rendering index, color fidelity index, light-emitting diodes, lowcost, natural products, green chemistry

Light-emitting diodes (LEDs) are a lighting technology with a huge and ascending market.

Typically, LED backlights are often paired with inorganic phosphors made from rare-earth elements (REEs) to tune the emission lineshapes for different applications. However, REE production is a resource-intensive process with many negative environmental impacts. Herein we develop organic hybrid LEDs using organic dyes synthesized from an abundant and nontoxic natural product (theobromine) to replace REE phosphors. The resulting hybrid LED generates continuous emission from $400-740 \mathrm{~nm}$, resulting in high color rendering index (the current industry standard) of 90 and color fidelity index (the most advanced and comprehensive standard) of 92, challenging commercial LEDs based on REE phosphors. In addition, the light-converting composite is made from $99 \mathrm{wt} \%$ SBS, an inexpensive industrial polymer, and $1 \mathrm{wt} \%$ theobromine dyes, reducing the cost of the light converter to $\phi 1.30$ for a 1 W LED, compared to approximately $₫ 19.2$ of commercial products. The light converting efficiency of the dye-SBS composite is $82 \%$. Excited state kinetics experiments were also conducted to provide guidance to further increase the light-converting efficiency of the theobromine dyes while maintaining excellent color rendering and fidelity. 


\section{Introduction}

Light-emitting diodes (LEDs) are a rare-earth element (REE) based technology with surging market demand ${ }^{[1]}$ REEs are used in the production of phosphors, a light converter in LEDs to tune their emission spectra based on the needs of different lighting applications. Due to their high energy efficiency, 1,440 million units of REE-based LEDs were installed in the US till 2017, and this number is projected to reach 7,910 million by $2035 .{ }^{[2]}$ However, due to the necessity of mining and refining in REEs productions, environmental concerns such as deforestation, air pollution, and water contamination, have attracted substantial public scrutiny. ${ }^{[3]}$ In addition, REE supply is subject to future shortage considering their booming industrial demand and limited supply. ${ }^{[4]}$ Conversely, organic dyes are light-converting materials made from organic elements, primarily $\mathrm{C}, \mathrm{H}, \mathrm{N}, \mathrm{O}$, and $\mathrm{S}$, bypassing the need for REEs in light converter production. ${ }^{[5]}$ Therefore, the environmental profile of LEDs can be improved by adopting the organic hybrid LED architecture, switching the incumbent inorganic phosphor to organic dyes as shown in Figure 1. Furthermore, the scattering loss from inorganic phosphors can be eliminated due to the high transparency of organic dye composites. ${ }^{[1,6]}$

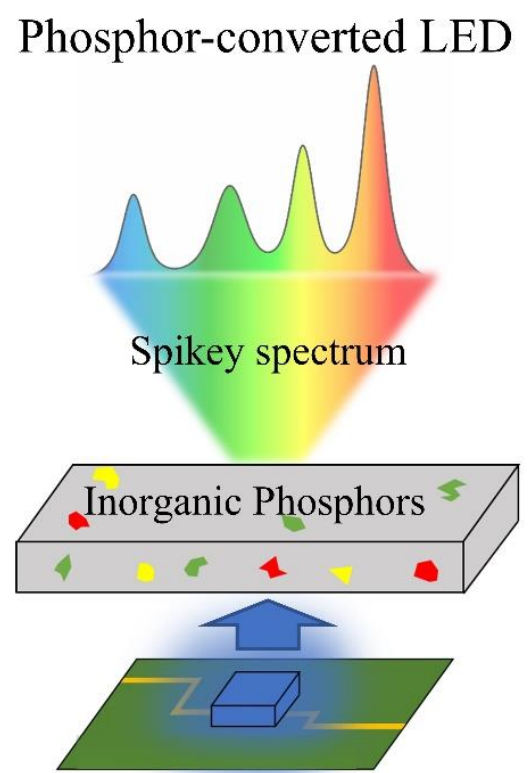

$450 \mathrm{~nm}$ LED

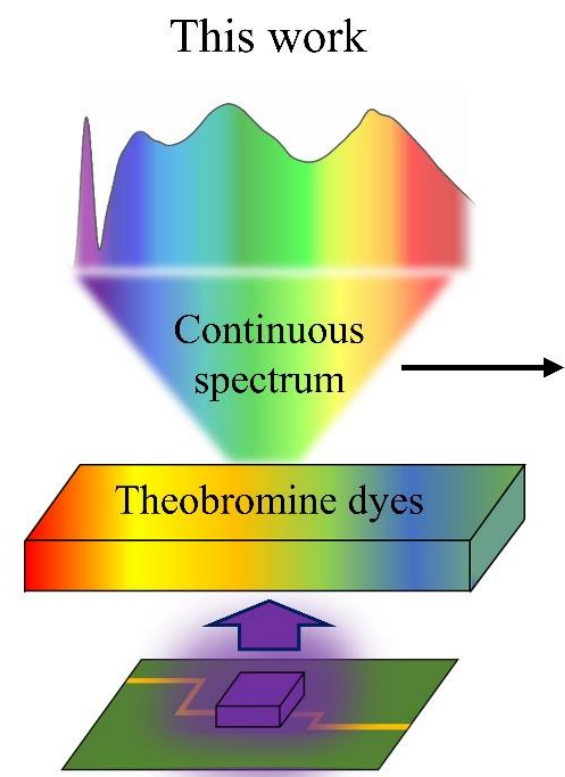

$405 \mathrm{~nm}$ LED
$94 \%$ visible wavelength coverage

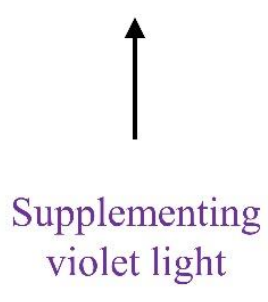

Figure 1. Structural comparison between commercial phosphor-converted LEDs and the organic hybrid LED proposed in this paper. Incumbent inorganic phosphors are powderous, introducing scattering loss and reducing the overall light output of the final LED. 
To gain industrial interest in organic dyes for LEDs, several issues must be addressed. Price plays a key role in the commercial viability of novel technologies. According to the U.S. Department of Energy, light converting materials typically account for around $24 \%$ of the total cost of a $1 \mathrm{~W}$ commercial warm-white LED ( $\varnothing 80)$, requiring the cost of the organic dye component to be less than $\varnothing 19.2 .^{[2]}$ However, the prices of industrially available fluorescent dyes are significantly higher than inorganic phosphors due to their complicated structures that require long synthetic routes and explosive and toxic reagents. ${ }^{[7]}$ In addition, the light converting materials should have high photoluminescent quantum yields (PLQYs) to improve the energy efficiencies of LED devices. For example, cerium doped yttrium aluminum garnet (YAG:Ce) is the most applied REE phosphor in LED lighting partially because it has PLQYs of around $90 \%{ }^{[8]}$

Currently most commercial LEDs pair yellow-emitting YAG:Ce phosphors with a blue backlight LED. The resulting white light LEDs achieve Color Rendering Indices (CRI, $R a$ ) near 80 of $100 .{ }^{[2]}$ However, the current industry CRI standard was first developed in the 1960s for the evaluation of fluorescent lights and as shown in Figure 2, $R a$ only evaluates 8 colors. ${ }^{[9]}$ Importantly, despite the fact that our brains weigh red more heavily than other colors, the 60s' CRI standard does not evaluate red color rendering. ${ }^{[10]}$ A good representation of red color is crucial not only in our daily lives (e.g. affecting our skin tones and increasing sales in department stores), but also in life-and-death moments (e.g. surgeries where it helps better distinguish different organs and blood vessels). Therefore, in updated CRI standards, an additional color rendering index for strong red, $R 9$, is supplemented, along with $R 10, R 11$, $R 12, R 13$, and $R 14$ for strong yellow, strong green, strong blue, light yellowish pink, and moderate olive green respectively. ${ }^{[9]}$ Considering that YAG:Ce is the most adopted light converter, and it has a limited emission in the red region, most commercial LEDs have $R 9$ values below 25. This explains why human skin looks pale in most LED lightings, despite their $R a$ of 80 . Therefore, both industrial and academic researchers are developing highperformance red phosphors to improve red emissions in LEDs. ${ }^{[2]}$

Still, the updated CRI standard is not ideal for assessing light source color rending. Due to the highly non-uniform color space of CRI, color differences are extremely exaggerated in the red region and suppressed in yellow and blue regions. ${ }^{[11,12]}$ Therefore, in 2015, a new evaluation system, TM-30, was developed by the Illuminating Engineering Society of North America that leverages a set of 99 real test samples with color space uniformity and spectral uniformity. ${ }^{[13]}$ In the TM-30 standard, there are two parameters to quantify the rendition of light sources. The Color Fidelity Index (CFI, Rf) quantifies the 
accuracy with which the color appearance of illuminated objects match their appearances under a reference light source (e.g daylight or black body radiation), and the color gamut index $(R g)$ quantifies the average increase or decrease of the chroma (the degree of vividness of colors) of objects related to those under reference illuminations.

\section{Color Samples for Color Rendering Index (e.g., $R a$ and $R 9$ )}

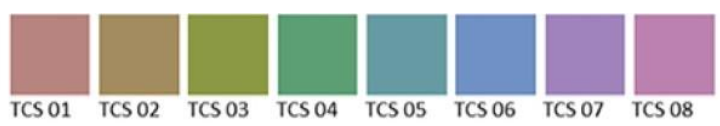

Color samples of CRI from 1960s

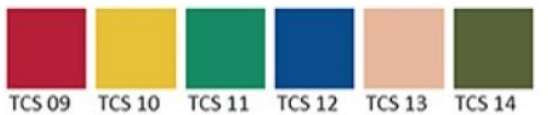

Strong colors supplemented in updated CRI

\section{Color Samples for Color Fidelity Index (e.g., $R \boldsymbol{f}$ and $\boldsymbol{R g}$ )}
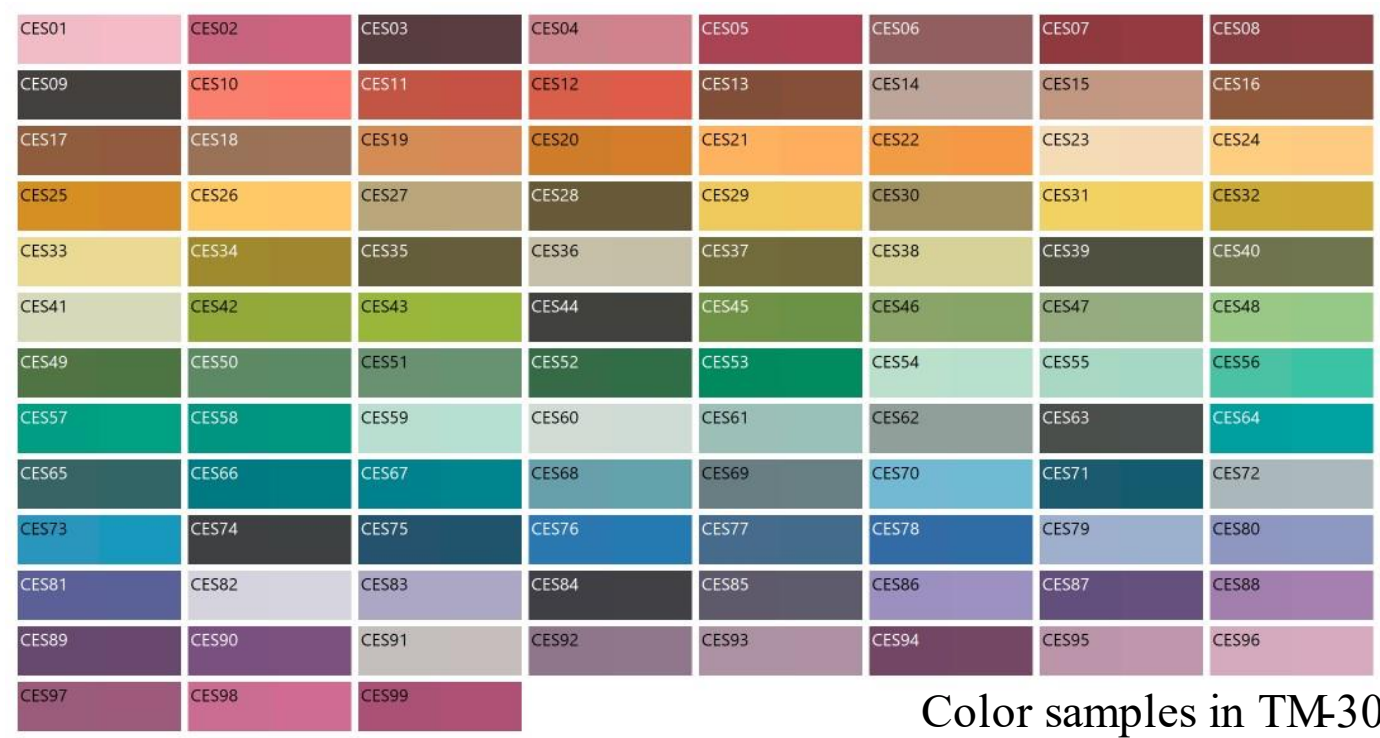

Color samples in TM-30

Figure 2. Demonstration of color samples of different methods utilized for lighting color quality evaluation.

Both sunlight and black body radiation possess continuous spectra across visible wavelengths. Therefore to achieve high $R f$ and $R g$ values in LED lightings, the phosphor emission spectra should be continuous and uniform across visible wavelengths. Using broadband emitters can simplify the manufacturing process by reducing the number of phosphors needed to achieve a uniform LED emission spectrum. Recently, our group reported several organic dyes with broad-band emissions from theobromine, an abundant natural product originally found in cacao beans. ${ }^{[7]}$ The syntheses of these theobromine dyes are streamlined and eco-friendly. The resultant dyes possess high PLQYs when blended with an inexpensive industrial polymer, poly(styrene-butadiene-styrene) (SBS), that serves as the host matrix to 
enable device integration. Moreover, these dye-SBS composites only require low blending ratios of theobromine dyes, around $1 \mathrm{wt} \% .{ }^{[14]}$ In this paper, we further advance the efficacy of theobromine dyes in LED lightings with a new deep red-emitting dye, Theo-Ruby. We developed a white light converter by blending Theo-Ruby with two other theobromine dyes (Theo-Blue and Theo-Green) into SBS. We paired the resulted light-converting dye-SBS composite with a $405 \mathrm{~nm}$ LED backlight to generate white emission (Figure 1). We further show that the final LED device fabricated with this combination of dyes could achieve a $R a$ of 90 and a $R f$ of 92 with continuous spectral coverage from $400-740 \mathrm{~nm}$, corresponding to $94 \%$ of visible wavelengths. This demonstrates that extending the emission to the violet region is crucial for high CFIs, in contrast to incumbent LEDs utilizing a $450 \mathrm{~nm}$ LED backlight. Moreover, the dyes' low blending ratios significantly reduce the estimated cost of the light converting film to $\varnothing 1.30$ for a $1 \mathrm{~W}$ LED, which is significantly lower than the estimated cost of incumbent inorganic phosphor-converted LEDs.

\section{Results and Discussions}

Scheme 1 shows the synthetic routes to converting alkylated theobromine into TheoBlue, ${ }^{[7]}$ Theo-Green ${ }^{[14]}$ and Theo-Ruby. In the molecule design, alkylated theobromine increases PLQYs of the final products and their solubility in SBS, ${ }^{[7]}$ while the central $\pi$ conjugated luminophores are used to tune their emission wavelengths. These two key components guide the synthesis of theobromine dyes of various emission wavelengths and enhanced PLQYs. Theo-Blue, Theo-Green and Theo-Ruby were synthesized by crosscoupling alkylated theobromine with pyrene, benzo[c] $[1,2,5]$ thiadiazole and naphtho[2,3c] $[1,2,5]$ selenadiazole, affording organic dyes of desired emissions respectively in blue, green and red regions required for high CRI and CFI lightings. It is worth mentioning that the introduction of selenium atom into Theo-Ruby is necessary to achieve sufficient emission in the strong-red and deep-red region (beyond $610 \mathrm{~nm}$ ) to realize continuous emission across visible wavelengths. It was found that the light-converter fabricated from its sulfur counterpart (Theo-Red) only achieved $R a$ of 70 due to limited spectral coverage beyond 610 $\mathrm{nm} .^{[14]}$ This highlights the excellent structural and spectral tunability of organic dyes. 

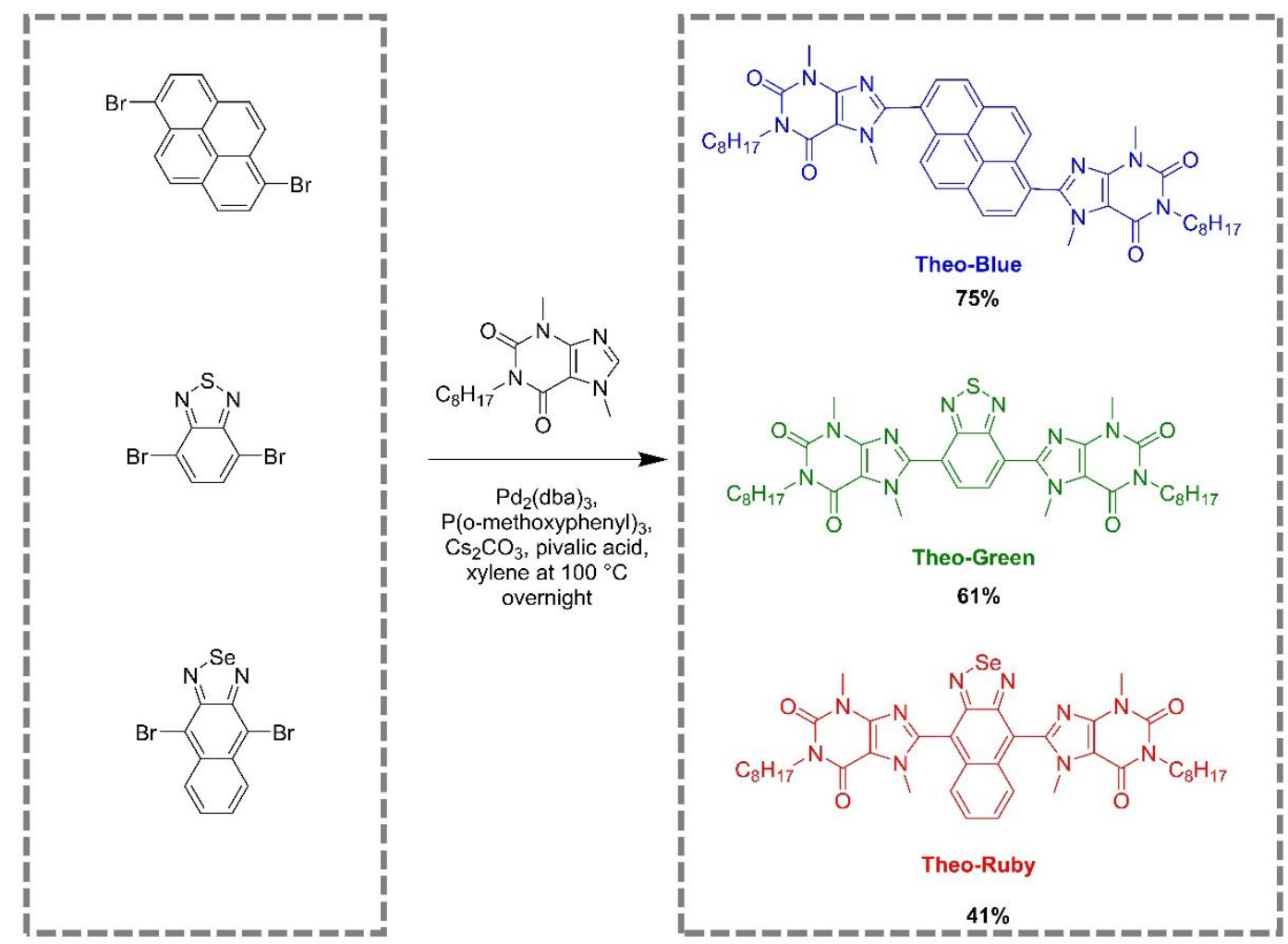

Scheme 1. Synthetic route for Theo-Blue, Theo-Green and Theo-Ruby.

In our syntheses, direct arylation was applied to cross-couple alkylated theobromine with the chosen luminophores, instead of Stille or Suzuki coupling commonly applied in organic dye syntheses. Direct arylation is an ascending green and atom-efficient methodology for synthesizing aromatic compounds. ${ }^{[15]}$ It directly utilizes a C-H bond for C-C bond formation, and bypasses C-H pre-functionalization required for Stille or Suzuki coupling. This not only shortens the synthetic routes, but also avoids utilizing toxic reagents (e.g., organotin) and explosive reagents (e.g., organolithium) commonly used in $\mathrm{C}-\mathrm{H}$ bond prefunctionalization. Their streamlined syntheses restrict their production cost to a relatively low range - the cost of Theo-Blue, Theo-Green and Theo-Ruby are respectively $\$ 14.6 / \mathrm{g}, \$ 17.1 / \mathrm{g}$ and $\$ 45.6 / \mathrm{g}$ based on lab-scale estimation (detailed cost breakdowns are shown in SI Section 2). The price of rare-earth phosphors was estimated based on the cost of a $1 \mathrm{~W}$ commercial warm-white LED ( $\varnothing 80)$ and the cost percentage (24\%) of rare-earth phosphors provided in a lighting report by the Department of Energy. ${ }^{[2]}$ This estimation is close to the procurement costs of lighting manufacturers. On the other hand, our cost calculations of theobromine dyes based on lab-scale synthesis may underestimate cost savings from bulk ordering and process optimization (e.g., solvent recycling) in industrial production. ${ }^{[16]}$ More importantly, the final 
LED light-converting film only requires around $1 \mathrm{wt} \%$ of the theobromine dyes to be blended into an inexpensive industrial polymer, SBS (purchased from Sigma Aldrich at $\$ 82.4 / \mathrm{kg}$ ), and thus the overall cost contribution from the theobromine dyes is minimal, as discussed in a previous report by our group. ${ }^{[14]}$.

To combine these dyes with a $1 \mathrm{~W} 405 \mathrm{~nm}$ LED, the light converting composite is made by dissolving $50 \mathrm{mg}$ SBS, $0.5 \mathrm{mg}$ Theo-Blue, $0.025 \mathrm{mg}$ Theo-Green and $0.025 \mathrm{mg}$ Theo-Ruby into $0.5 \mathrm{ml}$ toluene and drop casting the resultant solution onto a clean glass substrate. While the cost of the $405 \mathrm{~nm}$ LED is comparable to that of $450 \mathrm{~nm}$, the cost of our light converting film is $\varnothing 1.30$, which is significantly lower than $\varnothing 19.2$ for a comparable LED phosphor fabricated with REE phosphors. ${ }^{[2]}$ Notably, the blending ratios of Theo-Green and Theo-Ruby are only $10 \%$ of Theo-Blue's. We attribute this to Theo-Green and Theo-Ruby both absorbing light at around $405 \mathrm{~nm}$, and the emission of Theo-Blue overlapping with the absorption of Theo-Green and Theo-Ruby (Figure 3). Therefore, within the composite, a portion of the blue light generated by Theo-Blue is reabsorbed by Theo-Green and Theo-Ruby and converted to green and red light, respectively. Similarly, the green light generated by Theo-Green is reabsorbed by Theo-Ruby. We do not believe that energy transfer is taking place between the theobromine dyes since the distance between each molecule is calculated to be around $10 \mathrm{~nm}$ (estimated based on the blending ratio of $1 \mathrm{wt} \%$ ), which would be too large for energy transfer to take place. ${ }^{[17]}$

Figure 3 shows the absorption and photoluminescence spectra of Theo-Blue, TheoGreen, and Theo-Ruby blended in SBS at $1 \mathrm{wt} \%$. These dye-SBS composites have strong absorption from $300-420 \mathrm{~nm}$, which enables the use of a violet excitation backlight (e.g. 405 $\mathrm{nm}$ used in this paper). Their emissions are respectively centered at $450 \mathrm{~nm}, 540 \mathrm{~nm}$ and 658 $\mathrm{nm}$. Notably, all three theobromine dyes have broad emission bands, with full-width half maximums (FWHMs) of $55 \mathrm{~nm}, 78 \mathrm{~nm}$ and $95 \mathrm{~nm}$. These large FWHMs are ideal for lighting applications because they enable wide spectral coverage of final LED devices. 

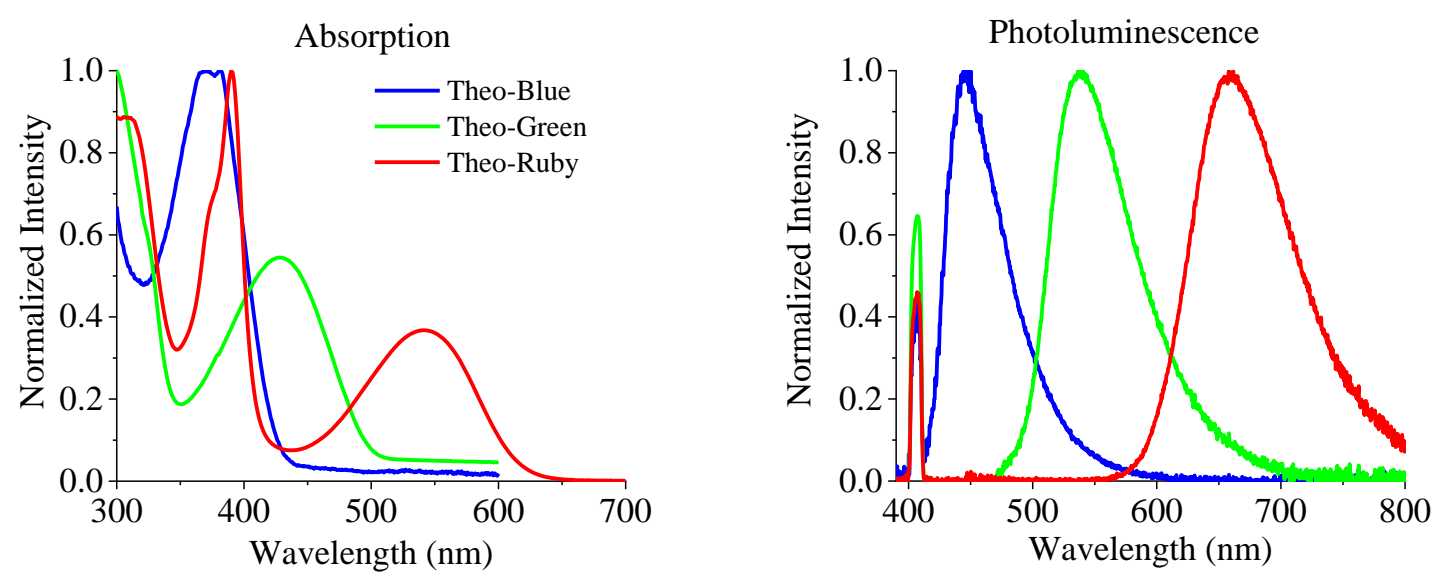

Figure 3. Absorption and photoluminescence spectra of Theo-Blue, Theo-Green and TheoRuby in SBS at $1 \mathrm{wt} \%$ (the narrow peak at $405 \mathrm{~nm}$ is attributed to unfiltered excitation light).

Figure 4a shows the emission spectra of the hybrid LED device, utilizing a $1 \mathrm{~W} 405$ nm LED chip as the light source and the light-converting dye-SBS composite. Due to the balanced intensity of violet, blue, green and red light, the emission of the hybrid LED achieved a CIE coordinate of $(0.34,0.34)$ and a correlated color temperature of 5383K (Figure $4 \mathrm{c} \& 4 \mathrm{e})$. This emission deviates little from the equal-energy white light standard, whose CIE coordinate is $(0.33,0.33)$, or the black body radiation curve with deviation $\left(D_{u v}\right)$ as small as 0.0003. Figure $4 \mathrm{~d}$ shows the 14 special CRIs of the hybrid LED device. $R a$ and $R 9$ were respectively 90 and 71, which are significantly improved when compared to most common commercial LED lightings based on YAG:Ce ( $R a$ of 82 and $R 9$ of 24), as shown in Figure S2. Figure 4e \& 4f summarize the hybrid LED device's color renditions based on TM-30. A high $R f$ value of 92 is achieved, and all 99 special CFIs are larger than 80 . This indicates that when illuminated by a hybrid-LED device based on the theobromine dyes, the color appearance of illuminated objects matches well with their appearances under sunlight. The slightly higher $R g$ score of 104, compared to 100 of daylight, originates from the slight oversaturation at the blue and red region, as it is observed in Figure 4e. Figure $4 \mathrm{~g}$ shows the 99 special color fidelity indexes of a commercial specialty white LED with $R a$ of 97 and $R 9$ of 95. This commercial high-CRI LED uses a blue LED backlight (450 nm) and narrow-band converters. Unfortunately, due to the insufficient emission of the violet and blue wavelengths, it has lower special CFIs in both blue and purple regions (Figure $4 \mathrm{~g}$ ) compared to our devices with a $405 \mathrm{~nm}$ backlight (Figure 4f). Furthermore, in the green, yellow and red regions, there are some special CFIs lower than 80 , such as \#5, \#30,\#36, and \#38. This can be ascribed to 
the narrow emissions of inorganic phosphor components that cannot fully cover the visible spectrum. These two factors contribute to a relatively low $R f$ value of 89 , highlighting the advantages of utilizing a $405 \mathrm{~nm}$ backlight and broad-band emitters. In addition, the $R a$ and $R f$ values in our configuration are comparable to the state-of-art values from rare-earth phosphors and perovskite quantum dots down-converted LEDs that are currently under lab development (Table 1). In comparison to these reported structures, our materials possess economic and environmental advantages. Notably, there is a strong red emission peak in the emission spectrum of this high-CRI LED. This is crucial to obtain $R a$ and $R 9$ values approaching 100, whereas it does not lead to equally high $R f$ and high special CFIs at the red region. This underlines the disadvantages of the current industry CRI standard - it possesses a skewed color space where differences in red colors are exaggerated while differences in blue colors are suppressed. ${ }^{[11,13]}$

The overall efficiency of the hybrid LED is determined by the efficiency of the backlight LED that converts electricity into blue/violet light and the efficiency of the light converter that converts blue/violet light into longer wavelengths. PLQY is used to quantify the efficiencies of light converters and is defined by the ratio of the number of photons emitted by the light converter to the number of photons absorbed by the light converter. An integrating sphere was used to measure a PLQY of $82 \%$ for the white light converter, which is contradicted by the combined PLQY contributions from Theo-Blue ( 100\%), Theo-Green (94\%), and Theo-Ruby (39\%). This can be explained by the fact that the PLQY of Theo-Ruby is highly dependent on concentration due to the heavy atom effect from Se atoms, which introduces intersystem crossing (detailed in the following lifetime measurements) and reduces PLQY. Considering the blending ratio of Theo-Ruby in the white-light convertor is $0.05 \mathrm{wt} \%$, we measured the PLQY of $0.05 \mathrm{wt} \%$ Theo-Ruby-SBS blend, which is $66 \%$. It is worth mentioning that the PLQYs of Theo-Blue and Theo-Green are also reduced by this small amount of Theo-Ruby via the external heavy atom effect, ${ }^{[18]}$ which likely limits the PLQY of the white composite to $82 \%$. These results suggest that by changing the Theo-Blue:TheoGreen:Theo-Ruby ratio, one can adjust the blue, green, and red content in the emission spectra, thus tuning the color temperature. Because the PLQY of Theo-Blue and Theo-Green are similar, change in the Theo-Blue:Theo-Green ratio would not have a significant impact on the PLQY; however, the heavy atom effect from the Se in Theo-Ruby decreased the PLQY of the final dye-polymer composite. 


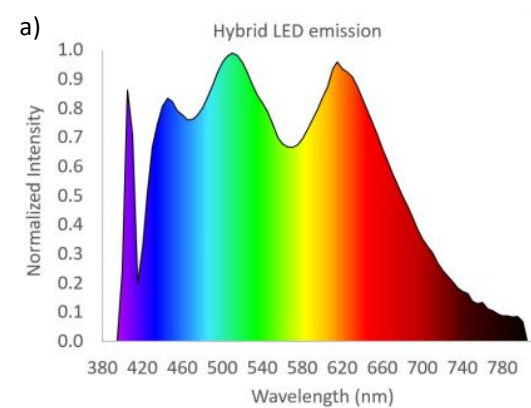

d)

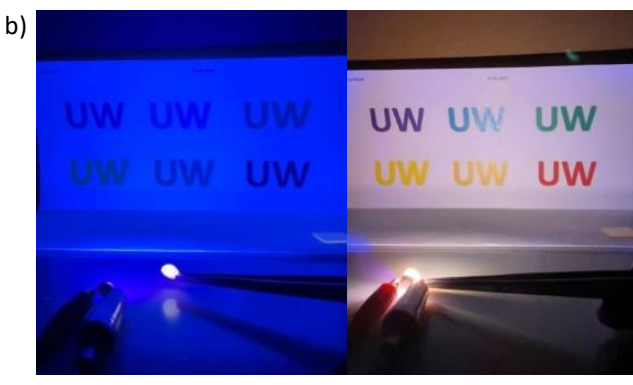

e)

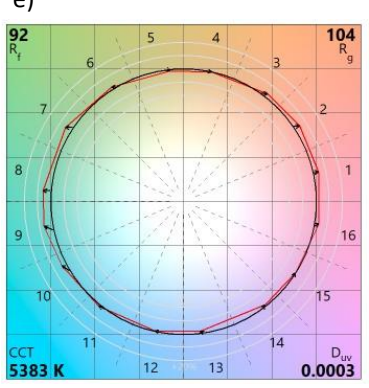

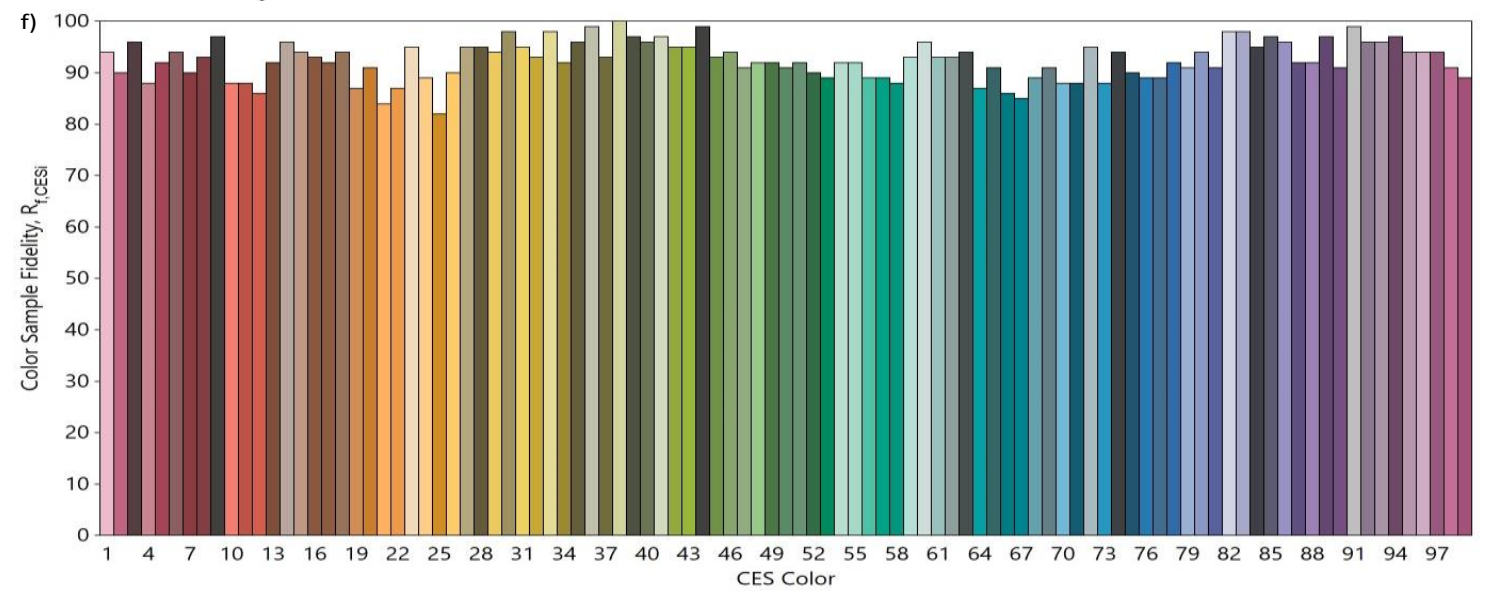

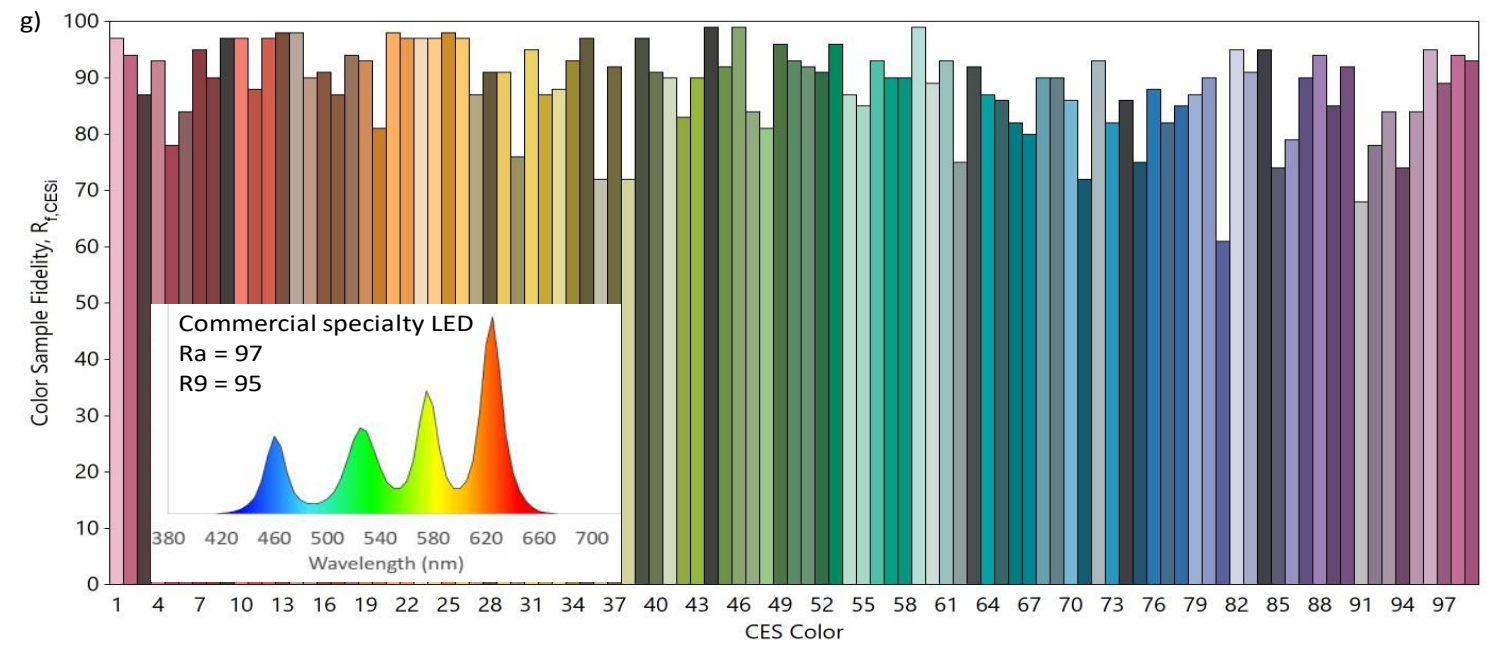

Figure 4. a) The emission spectrum of the hybrid LED with $405 \mathrm{~nm}$ blacklight and a theobromine dye SBS composite as a light converting layer; b) Photographs of a demonstration of light-conversion; c) CIE coordinate of the emission of the hybrid device; d) 14 special CRIs of the hybrid device; e) Color distortion graph: the hybrid LED device (red 
line) vs. standard white light (black line); f) 99 special CFIs of the hybrid LED; g) 99 special CFIs of the commercial high-CRI LED.

Table 1. Light quality rendition of different lighting configurations and PLQYs of their light converting materials.

\begin{tabular}{lcccccc}
\hline Category & PLQY & $R a$ & $R 9$ & $R f$ & $R g$ & Ref \\
\hline Theobromine dyes ${ }^{\text {a) }}$ & $82 \%$ & 90 & 87 & 90 & 104 & This work \\
Rare-earth phosphors & N.A. & 98 & 84 & 95 & 101 & ${ }^{[19]}$ \\
Rare-earth phosphors & N.A. & 95 & 95 & 91 & 102 & ${ }^{[20]}$ \\
Perovskite quantum dots & $61 \%$ & 96 & N.A. & 91 & 99 & ${ }^{[12]}$ \\
Carbon quantum dots ${ }^{\text {a) }}$ & $63 \%$ & 97 & N.A. & N.A. & N.A. & ${ }^{[21]}$ \\
Carbon quantum dots ${ }^{\text {a) }}$ & $48 \%$ & 86 & N.A. & N.A. & N.A. & ${ }^{[22]}$ \\
Carbon quantum dots ${ }^{\text {a) }}$ & $41 \%$ & 85.3 & N.A. & N.A. & N.A. & ${ }^{[23]}$ \\
\hline
\end{tabular}

a) Eco-friendly light-converters.

To better understand the origin of the lower PLQY of Theo-Ruby compared to other theobromine dyes, we conducted photoluminescence lifetime measurements on these dyes, and the results are summarized in Figure 5. To have a comprehensive understanding of the structure-PLQY relationship, Theo-Red (an analog to Theo-Ruby replacing the Se atom in Theo-Ruby to $\mathrm{S}$ atom) was also included. Theo-Red has a PLQY of $87 \%$ in SBS. ${ }^{[14]}$ Their prompt fluorescence lifetimes (Figure 5a) increase in the order of Theo-Blue (1.59 ns), TheoGreen (8.55 ns), Theo-Red (14.03 ns) and Theo-Ruby (14.6 ns). According to the Einstein relation, the lifetime of prompt transition is inversely proportional to the oscillator strength of the transition, and proportional to the square of the emission wavelength. ${ }^{[24]} \mathrm{We}$ observed a trend in the molecular orbital simulation of Theo-Blue, ${ }^{[7]}$ Theo-Green and Theo-Red ${ }^{[14]}-$ the spatial overlap between their highest occupied molecular orbitals and lowest unoccupied molecular orbitals decreases as the electron-withdrawing ability of the center unit increases (pyrene < benzo[c][1,2,5]thiadiazole < naphtho[2,3-c][1,2,5]thiadiazole). The reduced spatial overlap leads to a reduction in oscillator strength of the electronic transitions. The increasing values in emission wavelength and reducing values in oscillator strength together result in the increase of emission lifetimes in the order of Theo-Blue, Theo-Green to Theo-Red. The slightly longer lifetime of Theo-Ruby compared to Theo-Red can be explained by its slight redshift emission and small structure variation. Most of these lifetimes are monoexponential, but there is a longer lifetime component in Theo-Blue (12.85 ns) which may originate from the formation of pyrene excimers. ${ }^{[25]}$ 
Furthermore, in the $200-1000$ ns range of the lifetime plots (Figure 5b), the photon signal of Theo-Ruby is significantly stronger than those of the other theobromine dyes. The linear character of this signal on a log-log plot indicates that the lifetime decay follows a power law dependence at long times. This suggests that the long time decay of Theo-Ruby is ascribed to delayed fluorescence due to reversible trapping. ${ }^{[26,27]}$ We speculate that this stems from enhanced intersystem crossing (ISC) facilitated by the presence of heavy atoms, in this case, the Se atom in Theo-Ruby. ${ }^{[26]}$ Since ISC rate is proportional to $Z^{4},{ }^{[28]}$ it is likely that the ISC rate in Theo-Ruby is significantly higher than that of the other dyes. As shown in Figure 6, ISC converts singlet excited states into triplet excited states. Electronic transitions from triplet excited states to singlet ground states are usually slow and non-emissive due to spin restriction and their large energy gaps. Most of the triplet excited state electrons dissipate to the ground state through thermal decay instead of light generation, which is detrimental for high PLQYs. However, the energy offset between triplet excited states and singlet excited states is smaller. This smaller energy gap allows reversed ISC, back-converting non-emissive triplets to emissive singlets and enabling delayed fluorescence. Notably, both ISC and reversed ISC are still spin restricted, and they have much smaller kinetic rates compared to fluorescence, which is associated with transitions between singlet states. Therefore, we observed delayed fluorescence in smaller intensity and at longer lifetime ranges. These lifetime experiments demonstrate that the introduction of heavy atoms facilities non-radiative decay of theobromine dyes via ISC, which is a valuable insight for future development of theobromine dyes.
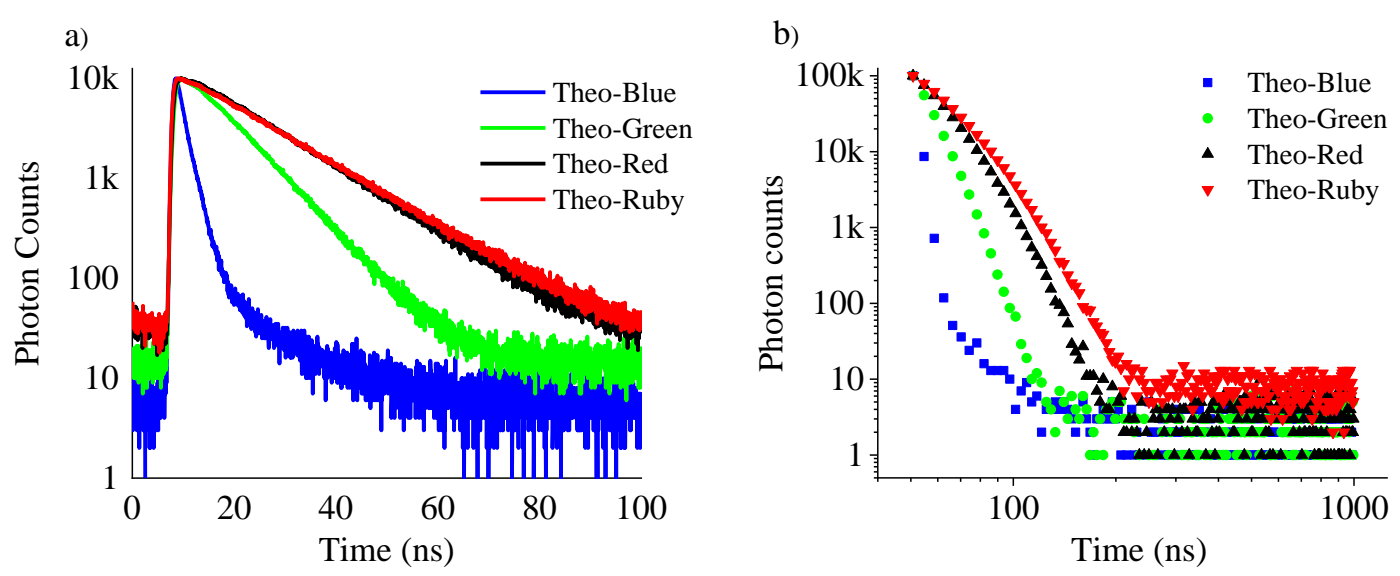

Figure 5. PL decay curves of theobromine dyes measured in an inert atmosphere with $405 \mathrm{~nm}$ excitation: a) short time range; b) long time range plotted on log axes. 


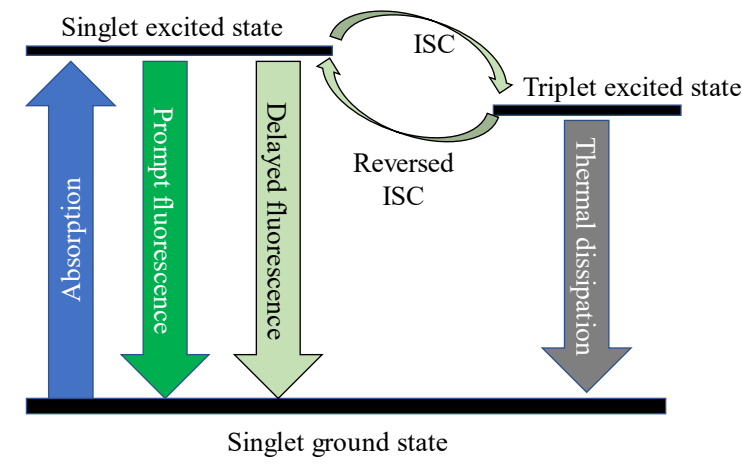

Figure 6. Energy level diagram to illustrate the interplay between singlet and triplet excited states via ISC and the resulting difference in prompt fluorescence (strong green) and delayed fluorescence (light green).

\section{Conclusion}

In this paper, we developed a hybrid LED structure utilizing three organic dyes (TheoBlue, Theo-Green and Theo-Ruby) synthesized from an abundant and non-toxic natural product (theobromine) with a $405 \mathrm{~nm}$ LED backlight. These three dyes possess broad emission and along with a $405 \mathrm{~nm}$ LED backlight, the resultant hybrid LED generates a continuous emission profile covering wavelengths from $400-740 \mathrm{~nm}$ (94\% of human visible wavelengths). This emission yields excellent color rendition values, $R a$ of 90 and $R f$ of 92 , which rival existing common LEDs and specialty LEDs in the market that are based on REE phosphors. In addition, the light-converting composite is made from $99 \mathrm{wt} \% \mathrm{SBS}$, an inexpensive industrial polymer, and $1 \mathrm{wt} \%$ theobromine dyes, reducing the cost of lightconverter to $\varnothing 1.30$ for a $1 \mathrm{~W}$ LED. This is significantly lower than the current cost of rareearth phosphors ( $\varnothing$ 19.2). The PLQY of the dye-SBS composite is $82 \%$, resulting from the combined contributions from Theo-Blue ( 100\%), Theo-Green (94\%) and Theo-Ruby (66\%). Their excited state kinetics shows that the reduced PLQY of Theo-Ruby stems from increased ISC due to the presence of selenium atom in the structure, providing guidance for future optimization of high-performance red theobromine dyes. 


\section{Acknowledgments}

The authors would also like to acknowledge the financial support from the Clean Energy Institute, NSF Partner for Innovation Award (2043422) (Y.H.), NSF Center for Selective CH Functionalization (CHE-1700982) (C.K.L.), and a Materials Research Science and Engineering Center (DMR-1719797) (T.A.C.). Y.H. thanks the Data Intensive Research Enabling Clean Technology (DIRECT) NSF National Research Traineeship (DGE-1633216) for support. Y.H. thanks Prof. Daniel R. Gamelin for support with stability measurements. We would like to thank and acknowledge the staff at the School of Pharmacy's Mass Spectrometry Center at the University of Washington for their instrumentation.

Received: ((will be filled in by the editorial staff))

Revised: ((will be filled in by the editorial staff)) Published online: ((will be filled in by the editorial staff))

\section{References}

[1] P. M. Pattison, M. Hansen, J. Y. Tsao, C R Phys. 2018, 19, 134.

[2] P. M. Pattison, M. Hansen, N. Bradsley, C. Elliott, K. Lee, L. Pattison, J. Tsao, 2019

Lighting R\&D Opportunities, https://www.energy.gov/sites/prod/files/2020/01/f70/ss1-rdopportunities2-jan2020.pdf, accessed: Dec, 2020.

[3] a) I. Razo, L. Carrizales, J. Castro, F. Díaz-Barriga, M. Monroy, Water Air Soil. Pollut. 2004, 152, 129; b) J. J. Swenson, C. E. Carter, J.-C. Domec, C. I. Delgado, PLOS ONE 2011, 6, 18875; c) L. J. Sonter, S. H. Ali, J. E. M. Watson, Proc. Biol. Sci. 2018, 285, 20181926.

[4] a) N. T. Nassar, X. Du, T. E. Graedel, J. Ind. Ecol. 2015, 19, 1044; b) K. S. Stegen, Energy Policy 2015, 79, 1.

[5] a) Y. Huang, D. L. Elder, A. L. Kwiram, S. A. Jenekhe, A. K. Y. Jen, L. R. Dalton, C. K. Luscombe, Adv. Mater. 2019, 1904239; b) X.-H. Zhu, J. Peng, Y. Cao, J. Roncali, Chem. Soc. Rev. 2011, 40, 3509; Y. Hong, J. W. Y. Lam, B. Z. Tang, Chem. Soc. Rev. 2011, 40, 5361; c) W. Li, Q. Liu, Y. Zhang, C. A. Li, Z. He, W. C. H. Choy, P. J. Low, P. Sonar, A. K. K. Kyaw, Adv. Maters. 2020, 32, 2001591.

[6] a) H. K. Park, J. H. Oh, Y. R. Do, Opt. Express 2012, 20, 10218; b) M. Nyman, L. E. Shea-Rohwer, J. E. Martin, P. Provencio, Chem. Mater. 2009, 21, 1536.

[7] Y. Huang, Y. Liu, P. J. W. Sommerville, W. Kaminsky, D. S. Ginger, C. K. Luscombe, Green Chem. 2019, 21, 6600. 
[8] H. M. Zhu, C. C. Lin, W. Q. Luo, S. T. Shu, Z. G. Liu, Y. Liu, J. T. Kong, E. Ma, Y. G. Cao, R. S. Liu, X. Y. Chen, Nat. Commun. 2014, 5, 4312.

[9] C. Li, M. R. Luo, C. Li, G. Cui, Color Res. Appl. 2012, 37, 160.

[10] A. Yoto, T. Katsuura, K. Iwanaga, Y. Shimomura, J. Physiol. Anthropol. 2007, 26, 373.

[11] D. Wendy, O. Yoshi, presented at Proc.SPIE, "Toward an improved color rendering metric", San Diego, California, United States, Sep. 2005.

[12] H. C. Yoon, J. H. Oh, S. Lee, J. B. Park, Y. R. Do, Sci.Rep. 2017, 7, 2808.

[13] A. David, P. T. Fini, K. W. Houser, Y. Ohno, M. P. Royer, K. A. G. Smet, M. Wei, L. Whitehead, Opt. Express 2015, 23, 15888.

[14] Y. Huang, T. A. Cohen, C. K. Luscombe, J. Mater. Chem. C 2021, DOI: https://doi.org/10.1039/D1TC01567B.

[15] a) Y. Huang, C. K. Luscombe, Chem. Rec. 2019, 19, 1039; b) T. Bura, J. T.

Blaskovits, M. Leclerc, J. Am. Chem. Soc. 2016, 138, 10056.

[16] F. Piccinno, R. Hischier, S. Seeger, C. Som, J. Clean. Prod. 2016, 135, 1085.

[17] S. Hartmann, D. Weidlich, D. Klostermeier, Methods in Enzymology, Academic Press, Cambridge, MA, USA 2016.

[18] Y. Shimizu, T. Azumi, J. Phys. Chem. 1982, 86, 22.

[19] Y. N. Ahn, K. D. Kim, G. Anoop, G. S. Kim, J. S. Yoo, Sci. Rep. 2019, 9, 16848.

[20] Y. Peng, H. Wang, J. Liu, Q. Sun, Y. Mou, X. Guo, ACS Appl. Electron. Mater. 2020, $2,2929$.

[21] Z. Wang, F. Yuan, X. Li, Y. Li, H. Zhong, L. Fan, S. Yang, Adv. Mater. 2017, 29, 1702910.

[22] L. Wang, W. Li, L. Yin, Y. Liu, H. Guo, J. Lai, Y. Han, G. Li, M. Li, J. Zhang, R. Vajtai, P. M. Ajayan, M. Wu, Sci. Adv. 2020, 6, 6772.

[23] Z. Wang, Y. Liu, S. Zhen, X. Li, W. Zhang, X. Sun, B. Xu, X. Wang, Z. Gao, X. Meng, Adv. Sci. 2020, 7, 1902688.

[24] A. Köhler, H. Bässler, Electronic Processes in Organic Semiconductors, Wiley, Hoboken, NJ, USA 2015.

[25] G. K. Bains, S. H. Kim, E. J. Sorin, V. Narayanaswami, Biochemistry 2012, 51, 6207.

[26] G. C. Hoover, D. S. Seferos, Chem. Sci. 2019, 10, 9182.

[27] A. Marchioro, P. J. Whitham, K. E. Knowles, T. B. Kilburn, P. J. Reid, D. R. Gamelin, J. Phys. Chem. C 2016, 120, 27040. 
[28] a) I. R. Gould, J. A. Boiani, E. B. Gaillard, J. L. Goodman, S. Farid, J. Phys. Chem. A 2003, 107, 3515; b) S. P. McGlynn, T. Azumi, M. Kasha, J. Phys. Chem. 1964, 40, 507; c) Z. Havlas, J. Michl, J. Am. Chem. Soc. 2002, 124, 5606.

Naturally derived organic dyes empower light-emitting diodes, providing color fidelity resembling sunlight.

Yunping Huang, Theodore A. Cohen and Christine K. Luscombe*

Naturally Derived Organic Dyes for LED Lightings of High Color Rendering and Fidelity Index

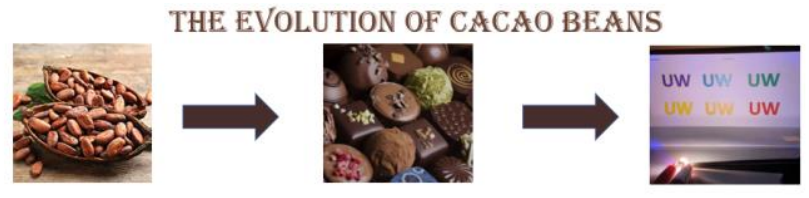

